\title{
THE APLYSIATOXINS: REACTIONS WITH ACID AND OXIDANTS
}

\author{
Y. KATO and P. J. ScheUER \\ Department of Chemistry, University of Hawaii, Honolulu, HI 96822, USA
}

\begin{abstract}
Under the influence of ethanolic acetic acid the aplysiatoxins rearrange with retention of all carbon atoms. An osmate of the anhydrotoxins can be reduced to a glycol which resists oxidative cleavage but loses water and rearranges with acid. Consideration of PMR and reactivity data lead to partial assignment of relative stereochemistry of the toxins.
\end{abstract}

\section{INTRODUCTION}

Sea hares are gastropod mollusks of the family Aplisiidae. Unlike many mollusks, which are familiar to us through their spectacular shells, these animals possess only a small internal shell or no shell at all. Sea hares have had some notoriety since ancient times because of their reputed toxicity. ${ }^{1}$ Early attempts by Flury ${ }^{2}$ and by Winkler and coworkers ${ }^{3}$ to characterize chemically or pharmacologically the toxic principle of Mediterranean ${ }^{2}$ or Californian $^{3}$ sea hare species were inconclusive. More recently Watson ${ }^{4}$ succeeded in isolating a toxic mixture from the digestive or midgut gland of four species of sea hares found on Hawaiian reefs. Watson ${ }^{5}$ further reported the separation of the sea hare toxin into a water-soluble fraction that possesses hypotensive activity, and into an ether-soluble entity with hypertensive properties. ${ }^{6}$ While Watson's work was not carried out with pure toxins, it defined the ether-soluble fraction sufficiently to allow us to purify this toxin for structural elucidation. We have reported part of this research in two previous papers, ${ }^{7,8}$ which dealt with chemical degradations in basic media and by reducing agents. The subject of this report will be transformations of the aplysiatoxins under oxidative and acidic conditions. ${ }^{9}$

Watson's so-called ether-soluble toxin, which we isolated from Stylocheilus longicauda (Quoy and Gaimard, 1824 ) in $0.025 \%$ yield and which has a lethality of $0.3 \mathrm{mg} / \mathrm{kg}\left(\mathrm{LD}_{100}\right.$, i.p. mouse) is a rather unstable viscous orange oil that proved to be an inseparable mixture of a nontoxic orange pigment and two colorless toxic components. Acetylation of the mixture furnished nontoxic diacetates, which could be chromatographically separated. Hydrolysis of the acetates on alumina under mild conditions $s^{10}$ proved that the structural integrity of the molecules had been preserved. In normal practice we carried out all subsequent work with the reasonably stable diacetates or even more conveniently, with the monomethyl ether monoacetates.

However, purification of the toxin via chromatography of the diacetates, or of the methyl ether esters, introduced a new complication. The two toxin derivatives suffered partial dehydration thus yielding a mixture of four rather than the anticipated two diacetates (or ether acetates). Apparently we were dealing with two isomeric pairs since the four compounds gave rise to only two sets of electron impact mass spectra. The true nature of this relationship was revealed only by chemical ionization mass spectrometry with ammonia as the carrier gas. Interestingly, dehydration occurred even when isobutane was the carrier gas.
Figure 1 shows the structure of aplysiatoxin (Ia, $R_{1}=$ $\mathrm{R}_{2}=\mathrm{H}$ ) and debromoaplysiatoxin $\left(\mathrm{Ib}, \mathrm{R}_{1}=\mathrm{R}_{2}=\mathrm{H}\right)$, which have a composition of $\mathrm{C}_{32} \mathrm{H}_{47} \mathrm{BrO}_{10}$ and $\mathrm{C}_{32} \mathrm{H}_{48} \mathrm{O}_{10}$ and differ from each other only by the bromine substituent in the aromatic ring. The phenolic hydroxyl is the abovementioned site of etherification (diazomethane) or acetylation and the aliphatic secondary alcohol is the other esterifiable group. The two artefacts, anhydroaplysiatoxin (IIa, $R_{1}=R_{2}=H$ ) and anhydrodebromoaplysiatoxin (IIb, $R_{1}=R_{2}=H$ ) arise from the toxins during chromatography (incompletely) or during electron impact mass spectrometry (completely) by facile loss of water between C-3 and $\mathrm{C}-4$, resulting in compositions of $\mathrm{C}_{32} \mathrm{H}_{45} \mathrm{BrO}_{9}$ and $\mathrm{C}_{32} \mathrm{H}_{46} \mathrm{O}_{9}$ for IIa and IIb.

\section{REACTION WITH ACID}

Our early attempts to achieve interpretable transformations of the aplysiatoxins by treatment with dilute mineral acid under various conditions always resulted in an unmanageable number of products. Our only successful reaction took place when debromoaplysiatoxin methyl ether acetate (III) was treated with acetic acid in ethanol $(1: 3)$ at room temperature for 7 days. In addition to the trivial product, anhydrodebromoaplysiatoxin methyl

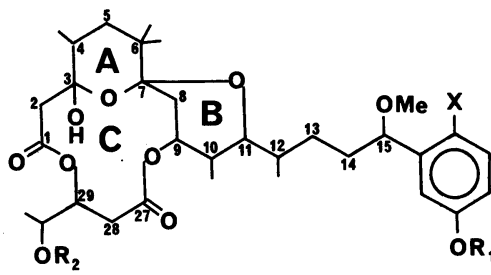

$$
\begin{array}{ll}
I_{\mathbf{a}} & \mathrm{X}=\mathrm{Br} \\
\mathrm{Ib}_{\mathbf{b}} & \mathrm{X}=\mathrm{H}
\end{array}
$$

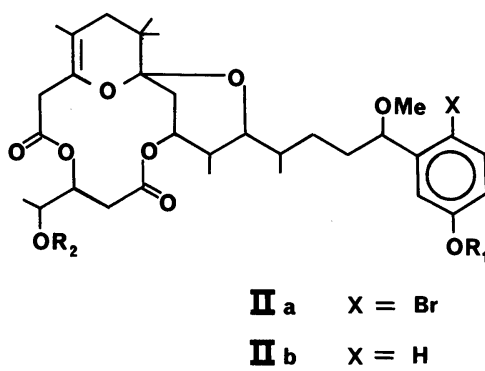

Fig. 1. Structures of the aplysiatoxins (I) and anhydroaplysiatoxins (II). 
ether acetate (IIb, $R_{1}=M e, R_{2}=A c$ ), which had merely suffered the familiar C-3, C-4 dehydration, we isolated two minor (uncharacterized) and one major new compound, an acid of composition $\mathrm{C}_{35} \mathrm{H}_{50} \mathrm{O}_{10}$, compared with a starting material formula of $\mathrm{C}_{35} \mathrm{H}_{52} \mathrm{O}_{11}$. Superficially, only a simple loss of water had occurred, but spectral data revealed that compound III had undergone a deep-seated series of transformations. For convenient handling of the rearranged product and in order to achieve ready chromatographic separation the carboxyl groups of the acidic products were esterified with diazomethane. It should be pointed out that we could not have elucidated this remarkable transformation product that contained all carbon atoms of the original toxins without the structural knowledge which we had gained from base catalyzed and reductive reactions described elsewhere. ${ }^{7,8}$

Figure 2 describes a plausible mechanistic pathway by which debromoaplysiatoxin methyl ether acetate (III) rearranges to the carboxylic acid VIIa, which we characterized as the methyl ester VIIb. Compound III, which is a hemiketal at C-3 and a ketal at C-7, may also be written as its diketoalcohol tautomer (IIIa). In the acidic reaction medium, intermediate IIIa, which is a $\delta$-diketone, may undergo a Knoevenagel condensation between anionic $\mathrm{C}-8$ and cationic $\mathrm{C}-3$, thus leading to $\beta$-hydroxyketone in a new carbocyclic six-membered ring (IV), which will spontaneously dehydrate to $\alpha, \beta$-unsaturated ketone $\mathrm{V}$. Enolization of the ketone group of $\mathrm{V}$ necessitates a shift of the $\alpha, \beta$-double bond to the $\beta, \gamma$-position, thus resulting

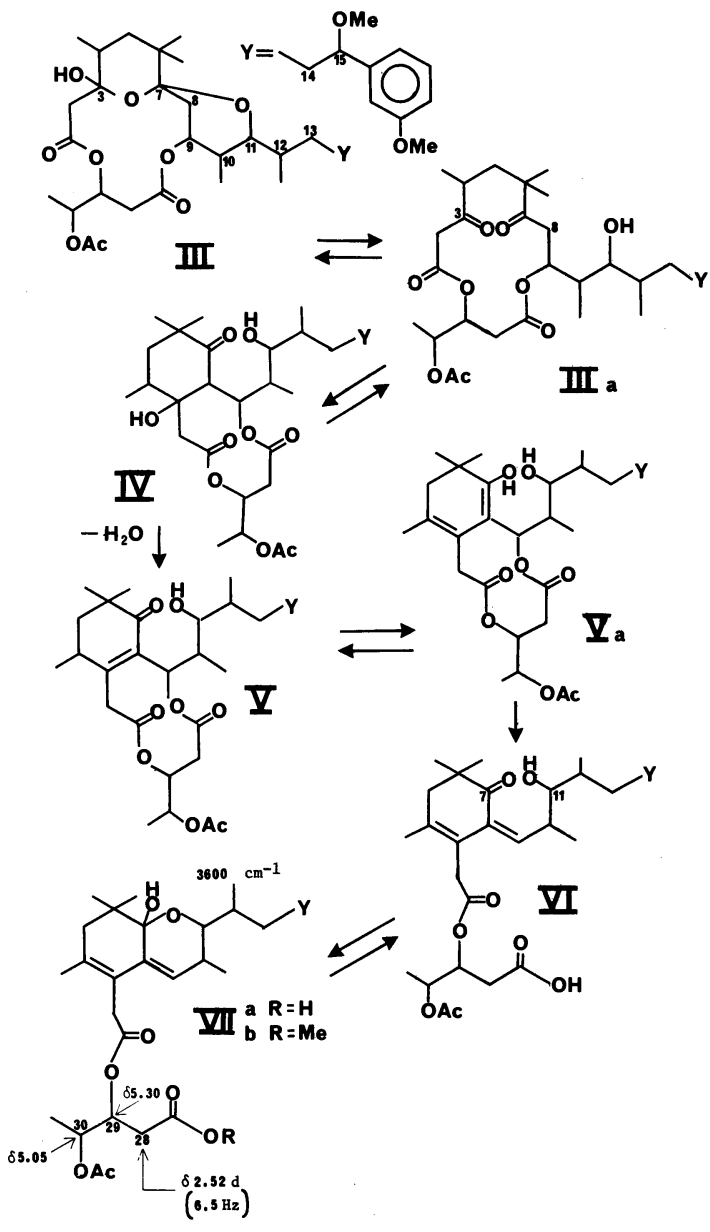

Fig. 2. Acid-catalyzed rearrangement of debromoaplysiatoxin methyl ether acetate (III). in dienol intermediate Va. Ketonization of the $\mathrm{Va}$ enol group will trigger the opening of the bis-lactone ring, which, with concomitant proton shift, will lead to the carboxylic acid VI. A plausible driving force for this last change may be relief from the constrained 10 -membered bis-lactone ring. It will be recalled that this ring was formed when the new carbocyclic 6-ring arose by internal condensation in the relatively strainfree 12 -membered bis-lactone of aplysiatoxin. Finally, the carbonyl group at C-7 is ideally situated to form a cyclic hemiketal (VIIa) by reacting with the hydroxy group at $\mathrm{C}-11$.

Our structural assignment rests on the following data. Broad infrared absorption at about $3600 \mathrm{~cm}^{-1}$ pointed to the presence in VIIb of an alcohol, which had to be tertiary and hindered since it failed to react with acetic anhydride/pyridine or acetic-formic anhydride/pyridine. Treatment of VIIb with acetic acid led to no further reaction, but allowing VIIa to stand in $0.5 \mathrm{M}$ hydrogen chloride in dry methanol led to complex decomposition products which we did not investigate. The only other outstanding feature of the infrared spectrum of VIIb was its carbonyl absorption at $1745 \mathrm{~cm}^{-1}$. The mass spectrum of VIIb was simple. It exhibited only three peaks which are interpreted in Fig. 3. An expected molecular ion at $m / e \quad 644$ is absent, but we see a respectable (14\%) M-18 peak which results from the dehydration of the tertiary alcohol. Compound VIIb, in common with other aplysiatoxin derivatives, invariably gives rise to a strong peak at $m / e 151$ arising from the benzyl portion of the molecule. The third peak at $m / e 419$ is logically derived from the $\mathrm{M}^{+}-18$ peak ( $m / e$ 626) by fragmentation which leads to a stable pyrylium ion. The following are key features of the PMR spectrum, which, when compared with spectra of the starting material and its congeners, suggested that the bis-lactone ring of aplysiatoxin had opened. A sharp two-proton doublet at $\delta 2.52(\mathrm{~J}=6.5 \mathrm{~Hz})$

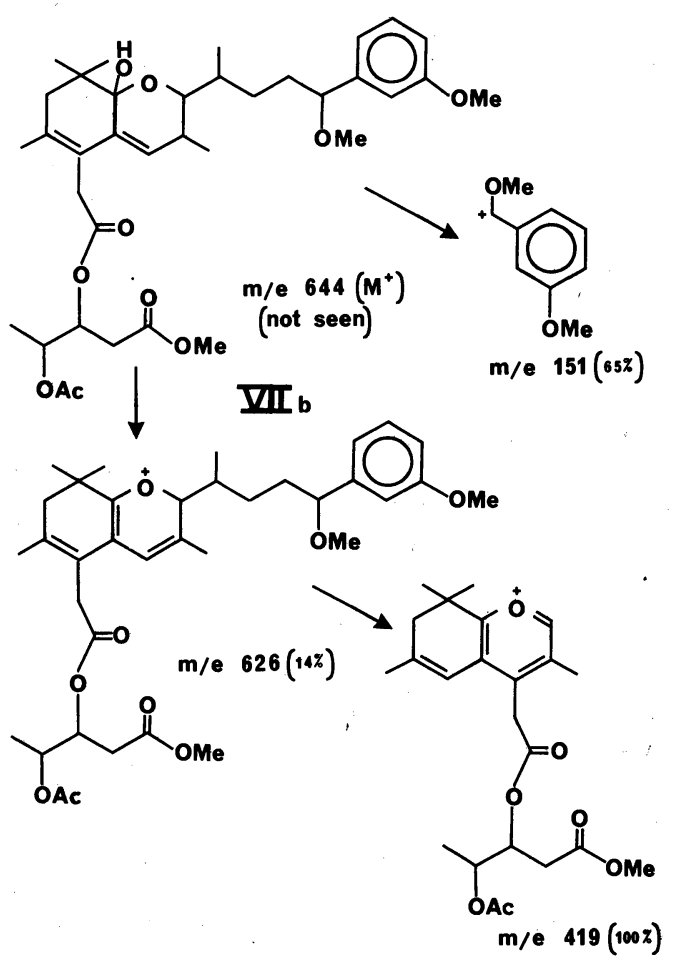

Fig. 3. Major mass spectral fragments of rearranged compound VIIb. 
assigned to the C-28 methylene group showed that this group was now acyclic. We further assigned two sharp and well-defined multiplets at $\delta 5.30$ and 5.05 to the methine protons at C-29 and C-30. A plausible alternate hemilactal formulation shown in Fig. 4 fails to explain the mass spectral data.

\section{OXIDATIONS}

We described in the Introduction the apparent isomerization of the aplysiatoxins (Ia, b, $R_{1}=R_{2}=H$ ) during chromatography, a transformation which we eventually recognized as a facile dehydration to the two anhydro compounds IIa and IIb $\left(R_{1}=R_{2}=H\right)$. The presumed composition of the toxins, $\mathrm{C}_{32} \mathrm{H}_{45} \mathrm{BrO}_{9}$ and $\mathrm{C}_{32} \mathrm{H}_{46} \mathrm{O}_{9}$, requires a structure with ten elements of unsaturation. Only nine of these could be unequivocally documented: four in the aromatic portion, two lactone carbonyls, one for the macro ring, and two for the spiropyran system. Presence of an additional ring appeared unlikely, but our evidence for an olefinic linkage in the aplysiatoxins was ambiguous at best. The PMR and i.r. spectra lacked signs of olefinic absorption; besides, CMR chemical shifts for two off-resonance singlets at $\delta 97.689$ and 99.939 (from TMS) seemed rather high-field and could not confidently be assigned to olefinic carbons. Eight carbon resonances at $\delta$ 112.316-169.558 had been firmly assigned to six aromatic and two carbonyl carbons.

The aplysiatoxins failed to reveal presence of a carboncarbon double bond under varied conditions of catalytic hydrogenation, ${ }^{9}$ which was also inconclusive since any carbon-carbon double bond in the toxins would be tetrasubstituted (no olefinic protons in PMR spectra) and no doubt rather hindered. We therefore attempted to settle the question of an olefinic linkage by oxidative transformations.

When anhydrodebromoaplysiatoxin methyl ether acetate (IIb, $R_{1}=\mathrm{Me}, \mathrm{R}_{2}=\mathrm{Ac}$ ) in ether/pyridine was stirred for $24 \mathrm{hr}$ at room temperature with an ethereal solution of osmium tetroxide, an osmate was formed, which could be isolated and purified by TLC, as evidenced by a small (6\%) but unambiguous molecular ion peak at m/e 884 for a composition of $\mathrm{C}_{35} \mathrm{H}_{50} \mathrm{O}_{14} \mathrm{Os}$. Reaction of the osmate with dilute aqueous sodium hydrogen sulfite in pyridine at room temperature furnished a single product after TLC purification. The i.r. spectrum of the presumed diol $\left(R_{1}=\right.$ $\mathrm{Me}, \mathrm{R}_{\mathbf{2}}=\mathrm{Ac}$ ) exhibited the anticipated hydroxyl bands at 3660 and $3500 \mathrm{~cm}^{-1}$ and should have structure VIII. A molecular ion was barely detectable at m/e 664 $\left(\mathrm{C}_{35} \mathrm{H}_{52} \mathrm{O}_{12}\right)$, and the compound could not be acetylated under normal conditions. These properties agreed with a diol which was derived from a tetrasubstituted double bond. The PMR spectrum confirmed that no other structural changes had taken place. Yet the diol proved to be

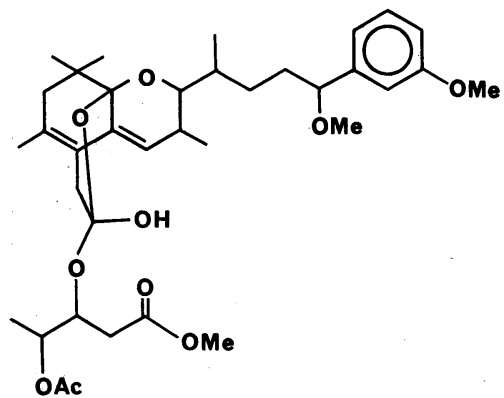

Fig. 4. Alternate structure for compound VIIb. unusual in that it failed to react normally with sodium metaperiodate or lead tetraacetate. A reaction did occur, loss of a mole of water, but it appeared that it was merely the acidic medium which had promoted the transformation. We confirmed this suspicion by realizing an identical dehydration reaction when we treated the diol with aqueous acetic acid-dioxane. The i.r. spectrum of the dehydration product (IX) clearly showed the absence of hydroxyl groups. The familiar ester absorption at $1730 \mathrm{~cm}^{-1}$ was present and there appeared to be a shoulder on the low frequency side of the carbonyl peak. Formulation of IX as the 3,4-epoxide seemed at first reasonable, but its i.r. spectrum lacked typical epoxide bands between 1250 and $750 \mathrm{~cm}^{-1}$. Furthermore, C-3 of this epoxide would also be a ketal carbon of somewhat doubtful stability. ${ }^{11,12}$ Loss of 28 mass units from the molecular ion $(m / e$ 646) suggested presence of a ketone, but one must bear in mind that epoxides are known to rearrange under electron impact. ${ }^{13,14}$ Nevertheless, compound IX may be rationalized to have arisen by ketonization of the hemiketal at C-3, followed by ketal formation between the hydroxyl at C-4 with the newly formed hydroxyl at C-7. On Vitride reduction compound IX is transformed to triol $\mathrm{X}$, which forms a diacetate and a triacetate under standard conditions. These reactions are outlined in Fig. 5, as is the complementary sequence which also leads to triol $\mathrm{X}$. Compound IIb may also be reduced with LAH to a $\mathrm{C}_{28}$-diol (XI), which we transformed via its osmate to a tetraol (XII), hence by acid to a ketodiol (XIII), which on

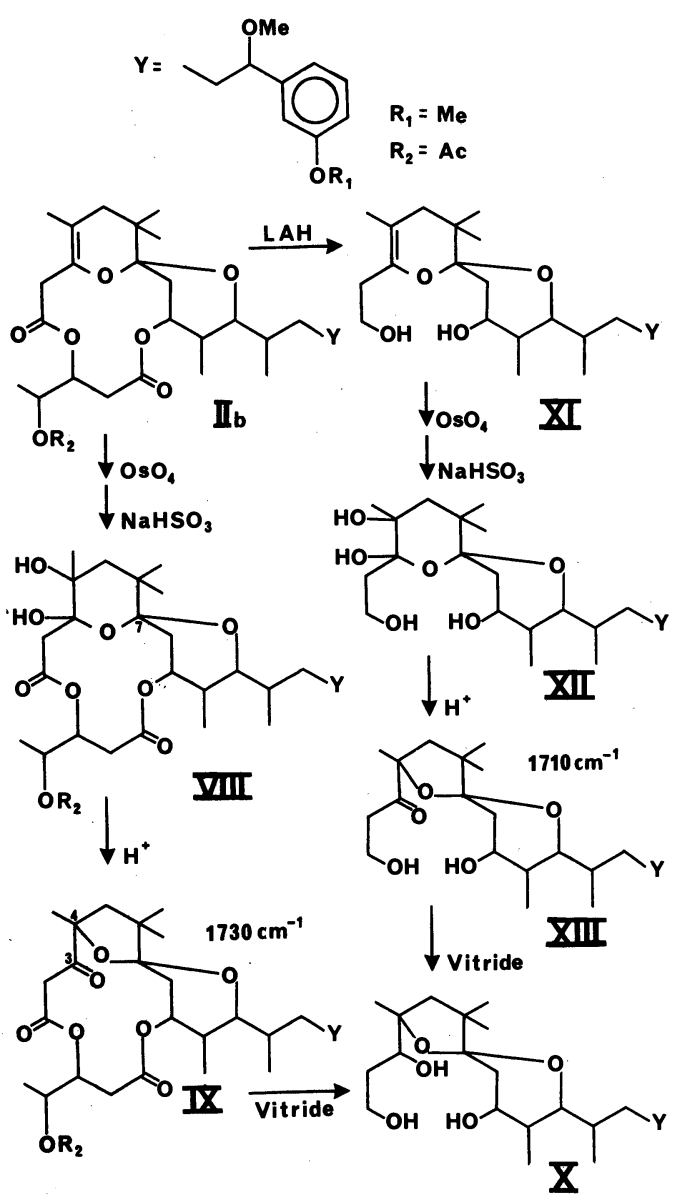

Fig. 5. Reaction of anhydrodebromoaplysiatoxin methyl ethe acetate with osmium tetroxide. 
Vitride reduction furnishes triol X. Compound XIII, in contrast to the analogous IX in the lactone series, shows an unencumbered carbonyl peak at $1710 \mathrm{~cm}^{-1}$. Mass spectral fragmentation of this entire series of compounds proved to be beautifully consistent and lent firm support to the formulation of the diol rearrangement to a ketoketal. All pertinent data are summarized in Fig. 6. These mass spectra are characterized by few peaks, of which $m / e 401$ proves to be the base peak, which is of equal intensity with the familiar peak at m/e 151 that constitutes a benchmark in all debromoaplysiatoxin derivatives.

An identical ketoketal (IX) was obtained when IIb $\left(R_{1}=\mathrm{Me}, \mathrm{R}_{2}=\mathrm{Ac}\right)$ was treated with ozone or with Jones reagent. Production of ketones from sterically hindered olefins by a wide range of oxidizing agents is not without precedent. An interesting series of analogous reactions was reported by Graham and Williams ${ }^{15}$ and is reproduced in Fig. 7.

\section{STEREOCHEMICAL CONSIDERATIONS}

Since in our hands no toxin derivative ever crystallized, we have no unequivocal X-ray diffraction data which would furnish full stereo structures of the aplysiatoxins. Analysis of PMR data and considerations of observed reactivities, however, permit us to make reasonable stereo-chemical assignments with considerable confidence.

Relative configuration of oxane ring $B$, which is part of the bis-oxaspiro system, is shown in Fig. 8. C-9 bears one hydrogen atom which resonates at $\delta 5.06$. It is a sharp quartet thus indicating that this proton is coupled to three vicinal hydrogens with nearly equal coupling constants of 2-3 Hz. Expected dihedral angles between $\mathrm{H}-9$ and its neighbors are $50-60^{\circ}$. Such a situation can be realized if $\mathrm{H}-9$ is equatorial and is part of a conformationally fixed six-membered ring. We were able to locate this characteristic quartet in many toxin derivatives at resonances from $\delta$ 4.85-5.24. Equatorial conformation of the pivotal proton at C-9 is confirmed by the following chemical evidence. Mild base treatment or mixed hydride reduction of the toxins eliminates the valeric acid fragment from the bis-lactone and allows isolation of oxyacids or diols as the case may be. ${ }^{9}$ These derivatives have in common a

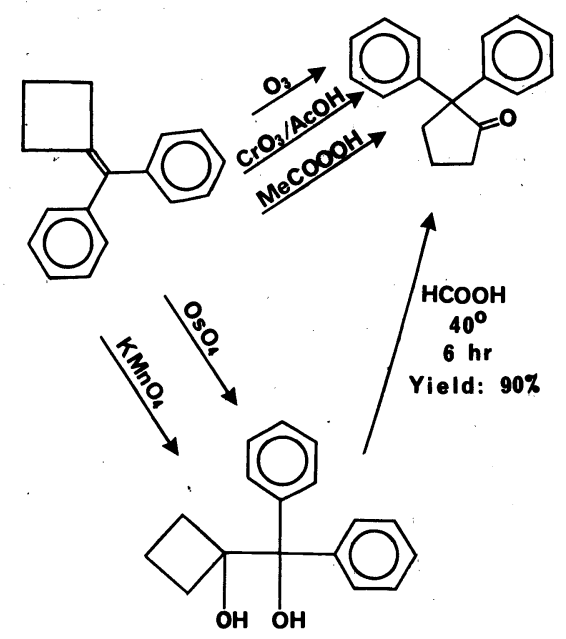

Fig. 7. Oxidative transformation of a hindered olefin. ${ }^{15}$

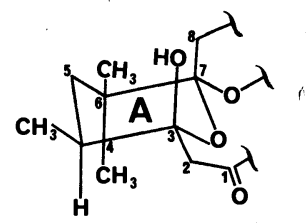

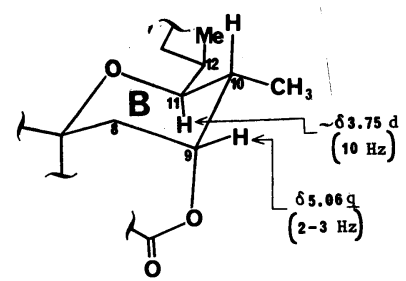

Fig. 8. Relative configurations and conformations of rings $A$ and B.

hindered hydroxy group, which can be slowly acetylated. This would be expected of an axial alcohol located on a conformationally rigid six-ring. ${ }^{16}$

The proton at $\mathrm{C}-11$ resonates as a broad doublet with a coupling constant of $10 \mathrm{~Hz}$ at chemical shifts of $\delta 3.65-$

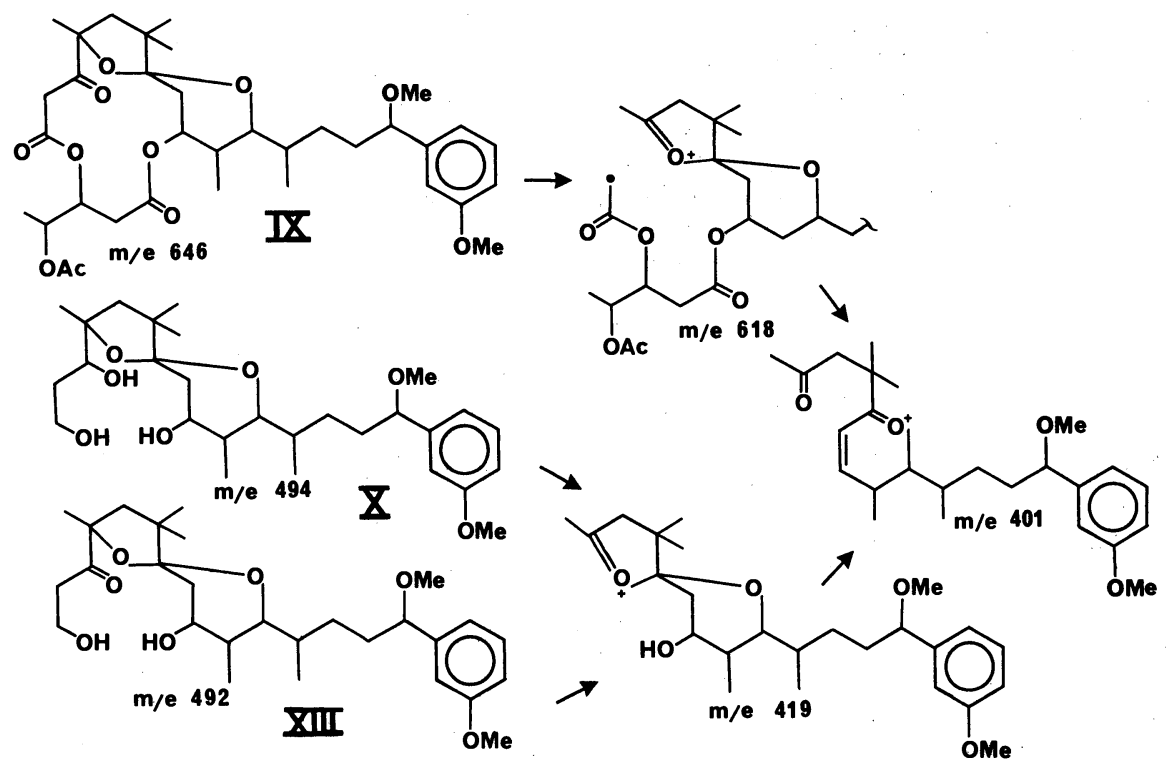

Fig. 6. Major mass spectral fragments of oxidation products IX, X and XIII. 


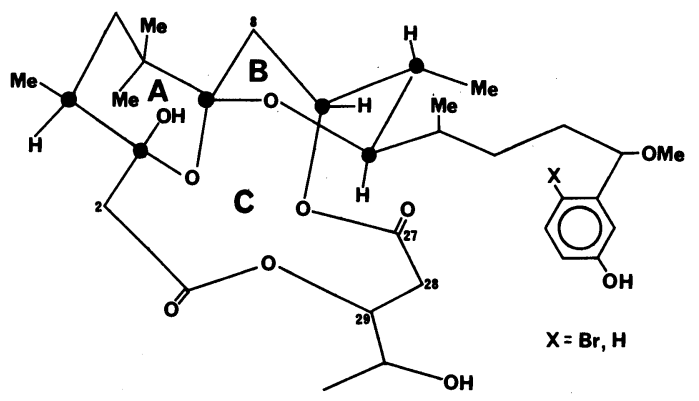

Fig. 9. Stereo structure of the aplysiatoxins.

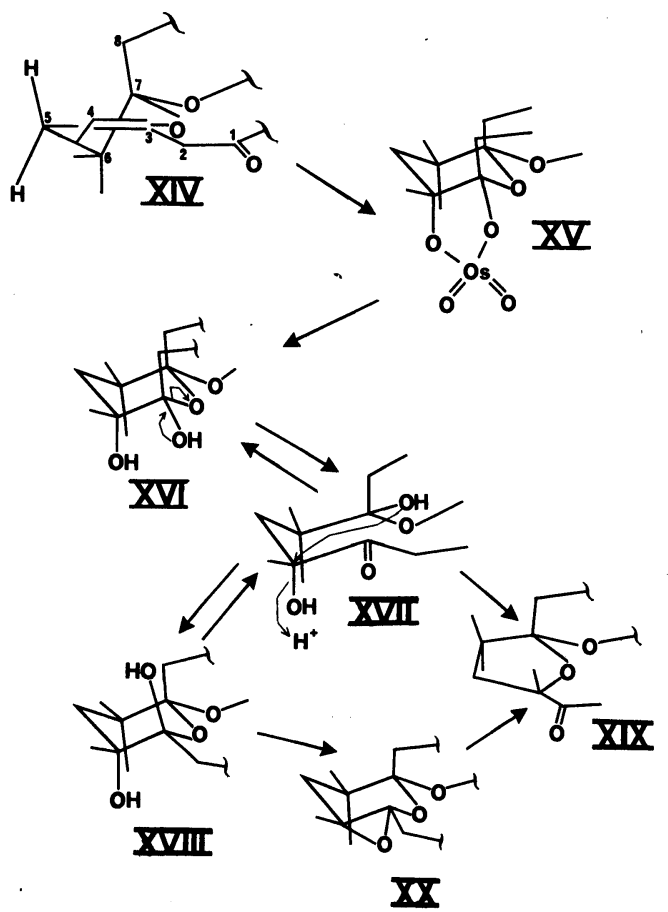

Fig. 10. Osmylation and further reactions of anhydrotoxin.

3.90 in a number of toxin derivatives. These data agree with trans diaxial conformation of $\mathrm{H}-11$ and the proton at $\mathrm{C}-10$. The weak coupling between $\mathrm{H}-11$ and the proton at $\mathrm{C}-12$ shows that rotation between $\mathrm{C}-11$ and $\mathrm{C}-12$ is rather restricted with a dihedral angle of $90^{\circ}$. Such an assignment is reasonable since this bond links the two large moieties of the toxin molecule.

When ring $\mathrm{B}$ is opened, as in some base transformation products, and the freed C-11 alcohol is acetylated, ${ }^{9} \mathrm{H}-11$ becomes a triplet at $\delta 4.77$ with a coupling constant of $6.0 \mathrm{~Hz}$.

If one constructs a Dreiding model of aplysiatoxin, the bis-lactone ring can be closed only when the carbonoxygen bond of ring $\mathrm{A}$ which originates at spiro carbon C-7 is axial to oxane ring B. Similarly, the C-7, C-8 bond of ring $B$ is axial to oxane ring $A$ on the basis of the following evidence. When the aplysiatoxins are converted to corresponding members of the anhydro series, the PMR resonanance of H-9 shifts upfield from $\delta 5.24$ to 4.85 . Since genesis of a C-3, C-4 double bond is not expected to alter conformation of ring $\mathrm{C}$ significantly, the observed shift may be attributed to anisotropic shielding by the new double bond. For such a shielding effect to operate, H-9 must lie above the plane of ring A, i.e. C-7, C-8 must be axial to ring $\mathbf{A}$.
With the stereochemistry about C-7 thus defined, ring A conformation follows readily. The C-2, C-3 bond must be equatorial to ring $\mathrm{A}$ in order to form ring $\mathrm{C}$ without 1,3-diaxial interaction. Conversely, the hydroxy group at C-3, which does suffer 1,3-diaxial interaction with the C-8 methylene, is axial and prone to undergo facile water elimination with the hydrogen at $\mathrm{C}-4$. These relationships are shown in Fig. 8. Conformation of the spiro bis-oxane system allows strainfree formation of ring C. Models indicate that the five atom sequence $0, \mathrm{C}-27, \mathrm{C}-28, \mathrm{C}-29$, $\mathrm{O}$ lies roughly perpendicular above the plane of ring $\mathrm{A}$. The entire aplysiatoxin molecule is shown in Fig. 9.

The stereochemical assignments of ring $A$ are further supported by the results of the osmium tetroxide oxidation, which are shown by part structures in Fig. 10. In the formation of the osmate XV from anhydrotoxin XIV the reagent is likely to attack from the backside of ring $A$ since frontal approach is blocked by the axial C-8 methylene group. Reductive hydrolysis of XV to cisglycol XVI is unexceptional, but ring $\mathrm{C}$ of glycol XVI may be somewhat strained because of 1,3-interaction between C-2 and C-8 methylenes. Release of this strain may be achieved by opening of ring $\mathrm{A}$ and changing the $\mathrm{C}-3$ hybridization from $\mathrm{sp}^{3}$ to $\mathrm{sp}^{2}$, analogously to the anomerization of a ketose. The resulting $\alpha$-ketol, intermediate XVII, is in equilibrium with the trans-diaxial glycol $\mathrm{XVIII}$, which is epimeric to cis-glycol XVI. This configuration explains the observed resistance of the glycol to periodate or lead tetraacetate cleavage. By acid, on the other hand, $\alpha$-ketol XVII may be dehydrated to the observed new ketal XIX ( $\equiv$ IX). One could also envisage the same product (XIX) to have arisen via trans-glycol XVIII and epoxide XX by elimination of the hindered tertiary hydroxy at C-3 with anchimeric assistance from the trans-hydroxy group at C-4.

Acknowledgement-We are grateful to Hoffman-La Roche, Inc. and to the U.S. Public Health Service for financial support.

\section{REFERENCES}

${ }^{1}$ B. W. Halstead, Poisonous and Venomous Marine Animals of the World. Vol. 1, p. 709 ff. U.S. Government Printing Office, Washington, D.C. (1965).

${ }^{2}$ F. Flury, Arch. Exp. Pathol. Pharmakol. 79, 250 (1915).

${ }^{3}$ L. R. Winkler, B. E. Tilton and M. G. Hardinge, Arch. Int. Pharmacodyn. Ther. 137, 76 (1962).

${ }^{4} \mathrm{M}$. Watson, Some aspects of the pharmacology, chemistry and biology of the midgut gland toxins of some Hawaiian sea hares, especially Dolabella auricularia and Aplysia pulmonica, Ph.D. Dissertation, University of HAwaii (1969).

${ }^{5}$ M. Watson, Toxicon 11, 259 (1973).

${ }^{6} \mathrm{M}$. Watson and M. D. Rayner, Toxicon 11, 269 (1973).

${ }^{7}$ Y. Kato and P. J. Scheuer, J. Am. chem. Soc. 96, 2245 (1974).

${ }^{8}$ Y. Kato and P. J. Scheuer, Pure Appl. Chem. 41, 1 (1975).

${ }^{9}$ Y. Kato, Toxic constituents of the marine mollusk Stylocheilus longicauda, Ph.D. Dissertation, University of Hawaii (1973).

${ }^{10}$ W. F. Johns and D. M. Jerina, J. Org. Chem. 28, 2922 (1963).

${ }^{11}$ R. N. McDonald, Rearrangements of $\alpha$-haloepoxides and related $\alpha$-substituted epoxides. In Mechanisms of Molecular Migrations. John Wiley (editor B. S. Thygarayan) p. 67. New York (1971).

${ }^{12}$ A. L. Mori, M. A. Porzio, and L. L. Schaleger, J. Am. chem. Soc. 94, 5034 (1972).

${ }^{13}$ H. Budzkiewicz, C. Djerassi and D. H. Williams, Mass Spectrometry of Organic Compounds. pp. 456, 459, Holden-Day, San Francisco (1967).

${ }^{14}$ Q. N. Porter and J. Baldas, Mass Spectrometry of Heterocyclic Compounds. p. 10 John Wiley, New York (1971).

${ }^{15}$ G. H. Graham and A. J. S. Williams, J. Chem. Soc. 4066 (1959).

${ }^{16}$ F. Sallman and Ch. Tamm, Helv. Chim. Acta 39, 1340 (1956). 